\title{
Please, do take it personally: an Exploration of Tailoring Health Messages
}

\section{Abstract}

Electronic- and mobile-health applications (Apps) are considered as potentially powerful means to improve the nutrition behaviour of users. In the design of App messages, "tailoring" can be used as it is found to be effective in affecting health behaviour outcomes including nutrition. Tailoring entails matching message content to characteristics of a message receiver. However, research provides some indications that message characteristics other than content could be related to the effectiveness of tailored communication (e.g. frequency). Furthermore, there are different approaches on how to execute tailoring. Two of such approaches are personalization and customization. With personalization, the preferences of a new App user are selected by a computer-tailoring-system based on the preferences of App users with similar characteristics. With customization, a new App user actively sets the preferences himself.

The aim of this research was to take a first step in investigating whether personalization and customization are suitable to tailor preferences for non-content message characteristics in the context of nutrition. Based on literature study and interviews with a number of dietitians $(n=3)$ a selection of personal characteristics and non-content message characteristics was made, and applied in the setup of a survey targeting respondents $(\mathrm{n}=37)$ with an interest in changing their dietary habits. The (Dutch) online survey was designed with the idea of asking respondents to enact setting up a new account with a mobile health App. So, respondents were asked to imagine that they were going to create an App account (NB they were told that it was a fictional account), and were asked to fill in some items regarding several personal characteristics. Next, respondents were asked to choose between setting preferences for the non-content message characteristics themselves (= customization) and setting preferences for non-content message characteristics of persons similar to themselves (e.g. gender and age) by the App (= personalization). Of the 37 survey respondents, most respondents $(73 \%, n=27)$ preferred customization in order to set their preferences in contrast to approximately a quarter of the respondents $(27 \%$, $\mathrm{n}=10)$ that preferred personalization.

In conclusion, this research found that at this moment the preferences of message receivers could be best tailored via customization due to a) great diversity in personal characteristics and preferences and b) a majority of the respondents that preferred customization over personalization. Nonetheless, it seems additionally worthwhile to further explore relations between personal characteristics and preferences to enable personalization as approximately a quarter of the respondents preferred this approach.

\section{Conflict of Interest}

There is no conflict of interest. 